\title{
THE PREVALENCE OF FAMILIAL HYPERCHOLESTEROLAEMIA IN PATIENTS WITH CLINICAL CORONARY ARTERY DISEASE
}

\section{J. Giovannucci1,2,3, H. Oo'1,2,3, D. Hare'1,2 R. O'Brien ${ }^{1,3}$ \\ ${ }^{1}$ The University of Melbourne, School of Medicine, Melbourne, Australia \\ ${ }^{2}$ Austin Health, Department of Cardiology, Melbourne, Australia \\ ${ }^{3}$ Austin Health, Department of Endocrinology, Melbourne, Australia}

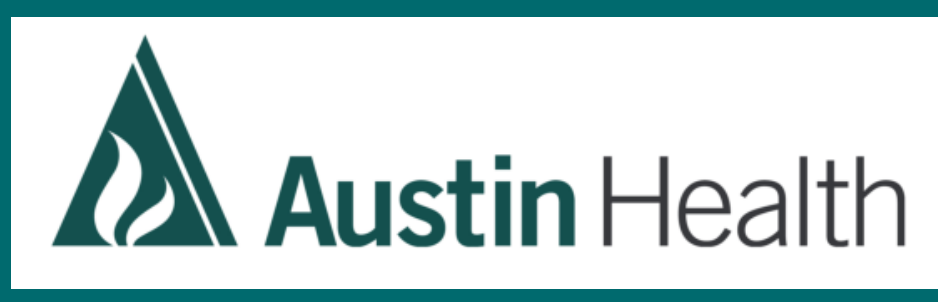

METHODS
BACKGROUND AND OBJECTIVES

\section{(MET}

- Cross sectional study, assessing patients $\leq 70$ yo at Austin Health, Melbourne, Australia from Feb-June 2018 presenting with:

- Acute coronary syndrome

- Elective percutaneous intervention

- Coronary artery bypass graft surgery

Diagnosis of $\mathrm{FH}$ was made utilising Dutch Lipid Clinic Network criteria

- "Definite" or "probable" FH if DLCN score > 5

- Cardiovascular disease risk factors: hypertension, diabetes mellitus, smoking status, elevated lipoprotein(a) were also recorded

- Wilcoxon rank-sum test and Fisher's exact test were used to analyse continuous and categorical variables

\section{RESULTS AND TABLES}

Table 1. Comparison of demographics and characteristics of patients diagnosed with $\mathrm{FH}$ (Definite/Probable $\mathrm{FH}, \mathrm{n}=19$ ) and patients without $\mathrm{FH}$ (Non-FH, $\mathrm{n}=158$ )

\begin{tabular}{|c|c|c|c|}
\hline & FH $n=19$ & Non-FH $n=158$ & $p$ value \\
\hline $\begin{array}{l}\text { Age in years, median (IQR) } \\
\text { Age less than } 60, n(\%)\end{array}$ & $\begin{array}{l}53(47-60.5) \\
14(73.7)\end{array}$ & $\begin{array}{l}61(57-67) \\
65(41.1)\end{array}$ & $\begin{array}{l}0.005 \\
0.013\end{array}$ \\
\hline BMI, median (IQR) & $28.7(26.6-35.6)$ & $29.1(26.5-32.8)$ & 0.821 \\
\hline $\begin{array}{l}\text { Pathology Results } \\
\text { Total Cholesterol in } \mathrm{mmol} / \mathrm{L} \text {, } \\
\text { median (IQR) } \\
\text { LDL in mmol/L median (IOR) }\end{array}$ & $5.1(4.9-5.9)$ & $4.0(3.2-5.1)$ & $p<0.001$ \\
\hline Lp(a) in g/L, median (IQR) & $0.62(0.19-0.96)$ & $0.23(0.10-0.54)$ & 0.040 \\
\hline Lp(a) greater than $0.5 \mathrm{~g} / \mathrm{L}, \mathrm{n}(\%)$ & $9(56.3)$ & 35 (29.9) & 0.048 \\
\hline $\begin{array}{l}\text { Lipid Lowering Medication use } \\
\text { Statin use at admission, } \mathrm{n}(\%)\end{array}$ & $18(94.7)$ & $100(63.3)$ & 0.004 \\
\hline $\begin{array}{l}\text { HBA1c as percentage, median } \\
(\mathrm{IQR})\end{array}$ & $5.8(5.6-7.4)$ & $5.8(5.4-6.8)$ & 0.411 \\
\hline Hypertension, $\mathrm{n}(\%)$ & $8(44.4)$ & $102(64.6)$ & 0.123 \\
\hline $\begin{array}{l}\text { Smoking status } \\
\text { Current Smoker, n (\%) }\end{array}$ & $8(44.4)$ & $51(32.9)$ & 0.431 \\
\hline
\end{tabular}

BMI, Body mass index; LDL, Low-density lipoprotein; Lp(a), Lipoprotein(a); HbA1c, Haemoglobin A1c; IQR, Interquartile range

Table 2. Comparison of $\mathrm{FH}$ prevalence, general demographics in patients aged less than $60(n=79)$ and those 60 and above $(n=98)$

\begin{tabular}{llll}
\hline & Age <60yo n=79 & Age 60+ $\mathbf{n = 9 8}$ & p value \\
\hline $\begin{array}{l}\text { Definite/Probable FH, } \\
\text { \%, (95\% CI) }\end{array}$ & $17.7(10.0-27.9)$ & $5.1(1.7-11.5)$ & $\mathbf{0 . 0 1 3}$ \\
BMI, median (IQR) & $28.7(26.6-34.8)$ & $29.3(26.5-32.1)$ & 0.575 \\
\hline $\begin{array}{l}\text { Pathology Results } \\
\text { Total Cholesterol in mmol/L, }\end{array}$ & $4.8(3.6-5.4)$ & $4.0(3.2-5.1)$ & $\mathbf{0 . 0 2 8}$ \\
$\begin{array}{l}\text { median (IQR) } \\
\text { LDL in mmol/L, median (IQR) }\end{array}$ & $2.9(1.8-3.6)$ & $2.1(1.4-3.0)$ & $\mathbf{0 . 0 0 4}$ \\
Lp(a) in g/L, median (IQR) & $0.30(0.13-0.78)$ & $0.20(0.10-0.54)$ & 0.390 \\
Lp(a) greater than 0.5 g/L, n (\%) & $20(35.7)$ & $24(31.2)$ & 0.709 \\
\hline $\begin{array}{l}\text { HBA1c as percentage, median } \\
\text { (IQR) }\end{array}$ & $5.6(5.4-7.5)$ & $5.8(5.4-6.8)$ & 0.803 \\
\hline Hypertension, $\mathrm{n}(\%)$ & $39(50.0)$ & $71(72.4)$ & $\mathbf{0 . 0 0 3}$ \\
\hline $\begin{array}{l}\text { Smoking status } \\
\text { Current Smoker, } \mathrm{n}(\%)\end{array}$ & $29(37.7)$ & $30(31.3)$ & 0.334 \\
\hline
\end{tabular}

$\mathrm{FH}$, Familial Hypercholesterolaemia; BMI, Body mass index; LDL, Low-density lipoprotein; Lp(a), Lipoprotein(a); HbA1c, Haemoglobin A1c; IQR, Interquartile range; $95 \% \mathrm{Cl}, 95 \%$ confidence interval
- 214 patients assessed during time period, 177 eligible for inclusion in analysis

- four patients had triglycerides $>4.5 \mathrm{mmol} / \mathrm{L}$ 11 patients had missing LDL-C levels, 21 patients were discharged before interview and one patient was non-English speaking

Estimated prevalence of $\mathrm{FH} 10.7 \%(95 \% \mathrm{Cl} 6.6 \%$ $-16.3 \%)$

- $\mathrm{FH}$ patients were younger, had higher LDL-C levels and higher rates of statin use

- 2/19 patients had a prior diagnosis of $\mathrm{FH}$

- $\quad$ 9/19 FH patients also had $L p(a)>0.5 \mathrm{~g} / \mathrm{L}$

Estimated prevalence in patients $<60 y$ o rose to $17.7 \%(95 \% \mathrm{Cl} 10.0 \%-27.9 \%)$

- $\mathrm{FH}$ prevalence in patients $60+$ was $\underline{5.1 \% \text {, }}$ (95\% Cl: $1.7 \%-11.5 \%) p=0.013$

\section{CONCLUSION}

- $\mathrm{FH}$ is over-represented but under recognised in this population

- Despite 18/19 FH patients being already treated with statins, a median LDL of 3.3 suggests suboptimal treatment of these patients

- Its high prevalence and associated morbidity and mortality makes a strong case for implementation of disease screening

Diagnosis also allows specialist lipidology referral and cascade screening of undiagnosed family members

\section{ACKNOWLEDGEMENTS}

I would like to thank Dr Hnin Oo for her assistance and Professor David Hare and Professor Richard O'Brien for their guidance in this project 